\title{
Extending States on Finite Concrete Logics ${ }^{1}$
}

\author{
Anna De Simone, ${ }^{2,5}$ Mirko Navara, ${ }^{3}$ and Pavel Pták ${ }^{4}$
}

Received November 10, 2003; accepted January 23, 2004

Published Online: August 23, 2007

\begin{abstract}
In this note we collect several observations on state extensions. They may be instrumental to anyone who pursues the theory of quantum logics. In particular, we find out when extensions (resp. signed extensions) exist in the "concrete" concrete logic of all even-element subsets of an even-element set (Th. 2.3 and Th. 2.9). We also mildly add to the study of difference-closed logics as initiated in Ovchinnikov (1999) by finding an extension theorem for subadditive states. Our results suplement the research carried on in De Simone (2000), Gudder (1979), Gudder and Marchand (1980), Navara and Pták (1983), Navara (1989), Ovchinnikov (1999), Pták (2000), Pták and Pulmannová (1991), Prather (1980), Sherstnev (1968), Sultanbekov (1992), and Svozil (1998).
\end{abstract}

KEY WORDS: (concrete) quantum logic; state; extension.

2000 AMS Classification: 06C15, 81P10.

\section{INTRODUCTION}

The question of extending states on quantum logics is sometimes surprisingly combinatorially involved. In spite of the progress made by the authors referred to in the abstract above, several questions remain open (see e.g. Ovchinnikov, 1999 and Pták, 2000). It therefore seems helpful to have the situation clarified in the "testing" case of the logic of even-number-element subsets of a set. This is what we intend to do in this note. Our results may partially overlap with the results of the previous effort but we are not aware of them being explicitly formulated elsewhere.

${ }^{1}$ The authors want to express their gratitude to the referee for valuable suggestions which corrected the original version.

${ }^{2}$ Dipartimento di Matematica e Statistica, Università Federico II di Napoli, Complesso Monte S. Angelo, Via Cintia - 80126 Napoli Italy; e-mail: annades@unina.it.

${ }^{3}$ Center for Machine Perception, Department of Cybernetics, Faculty of Electrical Engineering, Czech Technical University, Technická 2, 16627 Prague, Czech Republic; e-mail: navara@cmp.felk.cvut.cz.

${ }^{4}$ Department of Mathematics, Faculty of Electrical Engineering, Czech Technical University, Technická 2, 16627 Prague, Czech Republic.

5 To whom correspondence should be addressed; e-mail: annades@unina.it Originally published in International Journal of Theoretical Physics, Vol. 44, No. 7, 2005, Due to a publishing error, authorship of the article was credited incorrectly. The corrected article is reprinted in its entirety here. The online version of the original article can be found at http://dx.doi.org/10.1007/s10773-005-7083-6 


\section{NOTIONS AND RESULTS}

We shall exclusively deal with finite concrete (= set representable) quantum logics and states (= probability measures) on them as defined below. Standardly, for a set $X$ we stand $\exp X$ for the (Boolean) power algebra of $X$.

Definition 2.1. Let $X$ be a finite set. A collection $\Delta \subseteq \exp X$ is said to be a concrete quantum logic (abbr. a logic) if the following conditions are satisfied:

1. $X \in \Delta$;

2. if $A \in \Delta$, then $A^{c} \in \Delta\left(A^{c}\right.$ denotes the complement of $A$ in $\left.X\right)$;

3. if $A, B \in \Delta$ and $A \cap B=\emptyset$, then $A \cup B \in \Delta$.

Let $m: \Delta \rightarrow \mathbb{R}$ be a mapping from a logic into the set of real numbers. We say that $m$ is a signed measure on $\Delta$ if $m(A \cup B)=m(A)+m(B)$ provided that $A, B \in \Delta$ and $A \cap B=\emptyset$. A signed measure $m: \Delta \rightarrow \mathbb{R}$ is said to be $a$ state if $m(A) \geq 0$ for any $A \in \Delta$ and $m(X)=1$.

The chief question we ask here reads as follows: Having given a (concrete) $\operatorname{logic} \Delta$ on a set $X$ and having given a state $s$ on $\Delta$, when can we extend $s$ as a state (resp. as a signed measure) over the entire algebra exp $X$ ? Expressed more formally, given a state $s$ on $\Delta$, when can we find a state $t$ (resp., a signed measure $t$ ) on the Boolean algebra $\exp X$ such that $t$ restricted to $\Delta$ equals $s$ ? Let us observe first that certainly not always. In fact, we even do not have the "weak" extensions (= extensions of states to signed measures) of two-valued states at our disposal as the following simple example shows.

Example 2.2. Let $X=\{1,2, \ldots, 6\}$, and let $\Delta$ be the smallest concrete logic on $X$ containing the following four sets:

$$
A=\{1,2,3\}, \quad B=\{2,3,4\}, \quad C=\{3,4,5\}, \quad D=\{1,3,5\} .
$$

Obviously, $\Delta=\left\{\varnothing, A, A^{c}, B, B^{c}, C, C^{c}, D, D^{c}, X\right\}$ (for the reader acquainted with the theory of orthomodular lattices, $\Delta$ is a representation of the orthomodular lattice $\mathrm{MO}_{4}$ ). Let us take the (two-valued) state $s: \Delta \rightarrow\{0,1\}$ defined as follows:

$$
s(A)=0, \quad s(B)=1, \quad s(C)=0, \quad s(D)=1 .
$$

We claim that this state $s$ cannot be extended over $\exp X$ as a signed measure. Indeed, suppose that $m: \Delta \rightarrow \mathbb{R}$ is a signed measure which extends $s$. Then

$$
m(A)+m\left(B^{c}\right)+m(C)+m\left(D^{c}\right)=2 m(X),
$$

and, analogously,

$$
m\left(A^{c}\right)+m(B)+m\left(C^{c}\right)+m(D)=2 m(X) .
$$


But if in the left-hand side we replace $m$ by $s$, we obtain 0 in the first equality, and we obtain 4 in the second. A contradiction, thus, such an extension $m$ of $s$ does not exist.

It turns out, however, that many concrete logics do allow for the latter kind of extension. Such are for instance the logics of the following type (the conceptual value of these logics within various questions of quantum theories has been indicated in Gudder, 1979, Pták and Pulmannová , 1991, Svozil, 1998, etc.). Given a finite set $X$ of even cardinality, we denote by $X_{\text {even }}$ the concrete logic of all subsets of $X$ whose cardinality is even.

Theorem 2.1. Let $X$ be a finite set of an even cardinality. Let $s$ be a state on $X_{\text {even. }}$ Then $s$ can be extended as a signed measure over $\exp X$.

Proof: We can suppose that card $X \geq 4$ (otherwise, the result is trivial). Take an arbitrary triple $x, u, v$ of distinct elements of $X$. Let

$$
\bar{m}(x)=\frac{1}{2}(s(\{x, u\})+s(\{x, v\})-s(\{u, v\})) .
$$

We claim that $\bar{m}(x)$ is independent of the choice of $u, v$. Further, we claim that letting $x$ vary in $X$, we have defined a mapping $\bar{m}: X \rightarrow \mathbb{R}$ with the property that upon setting $m(A)=\sum_{a \in A} \bar{m}(a)$ for any subset $A$ of $X$, this $m$ constitutes a signed measure which extends $s$.

Let us first check that our definition of $\bar{m}$ is correct, i.e. let us show that $\bar{m}(x)$ does not depend upon the choice of $u, v$. Take first a couple $v, w$ such that $x, u, v, w$ are distinct. Let us show that both the couples $u, v$ and $v, w$ define the same $\bar{m}(x)$. Write

$$
f(x, u, v)=s(\{x, u\})+s(\{x, v\})-s(\{u, v\}) .
$$

We want to show that $f(x, u, v)=f(x, v, w)$. Let us compute the difference

$$
\begin{aligned}
f(x, u, v)-f(x, v, w)= & s(\{x, u\})+s(\{x, v\})-s(\{u, v\}) \\
& -s(\{x, v\})-s(\{x, w\})+s(\{v, w\}) \\
= & (s(\{x, u\})+s(\{v, w\}))-(s(\{x, w\})+s(\{u, v\})) \\
= & s(\{x, u, v, w\})-s(\{x, u, v, w\})=0 .
\end{aligned}
$$

Further, for a general element $t \in X$ distinct from $x, u, v, w$, we have $f(x, u, v)=$ $f(x, v, w)=f(x, w, t)$, proving independence of the value $\bar{m}(x)$ on the choice of $u$ and $v$. Let us check that $m$ defined as above extends $s$. Take a set $A=\{x, y\} \subseteq X$. Then we can find $u$ and $v$ such that $x, y, u, v$ are distinct and therefore 


$$
\begin{aligned}
m(A) & =\bar{m}(x)+\bar{m}(y)=\frac{1}{2}(f(x, u, v)+f(y, u, v)) \\
& =\frac{1}{2}(s(\{x, u\})+s(\{x, v\})-s(\{u, v\})+s(\{y, u\})+s(\{y, v\})-s(\{u, v\})) \\
& =\frac{1}{2}(2 s(\{x, y, u, v\})-2 s(\{u, v\}))=s(\{x, y\}) .
\end{aligned}
$$

The proof is complete.

Let us comment shortly on the previous result. Firstly, we cannot claim full novelty for this since in Prather (1980) it is obtained (for card $X>4$ ) as a consequence of a combinatiorial lemma of a broader bearing. We only demonstrate a rather straightforward way of showing the result. Secondly, in the logic $X_{\text {even }}$ we generally cannot extend states as states (see also Bhaskara Rao and Bhaskara Rao, 1983, Th. 3.5.1(v)).

Example 2.3. Let $X=\{1,2, \ldots, 2 k\}, k \in \mathbb{N}, k \geq 2$. Consider the state $s$ on $X_{\text {even }}$ such that $s(\{1, c\})=0$ and $s(\{b, c\})=\frac{1}{k-1}$ for all $b, c \in X \backslash\{1\}, b \neq c$. If $m$ is any signed measure on $\exp X$ extending $s$ we have $m(\{1\})=-\frac{1}{2(k-1)}$ and $m(\{c\})=\frac{1}{2(k-1)}$ for all $c \in X \backslash\{1\}$.

Secondly, observing that each two-valued state on $X_{\text {even }}$, with card $X \geq 6$, has to be a Dirac state (i.e. a state sitting in a point), we see that there must be extreme states on $X_{\text {even }}$ which are not two-valued (in fact, in Navara and Pták (1983) we have constructed some). And, thirdly, it is worthwhile observing that Th. 2.3 can also be proved, like many extension theorems which happen to hold, by the well-known criterion of Horn and Tarski (see e.g. Bhaskara Rao and Bhaskara Rao, 1983, Def. 3.2.1, 3.2.2, and Th. 3.2.5, 3.2.10). In the case of Th. 2.3 it would present another proof of a similar complexity. A minor advantage of the proof method used in Th. 2.3 is that it gives the result for group-valued measures for the groups which allow for dividing by 2 . Observe in passing that, for instance, the extension problem of coarse-grained measures as treated in Gudder and Marchand (1980) and Ovchinnikov (1999) finds in our opinion the Horn-Tarski criterion an effective proof device (see De Simone and Pták, 2006).

The logics of $X_{\text {even }}$ present a distinguished example of so-called differenceclosed concrete logics. The latter logics were introduced in Ovchinnikov (1999) under the name of symmetric concrete logics. In this note, let us use our notation (in our opinion more suggestive).

Definition 2.4. A concrete logic $\Delta$ on a set $X$ is said to be difference-closed if it is closed under the formation of symmetric differences, i.e., if for any couple of 
$A, B \in \Delta$ we have

$$
A \delta B=(A \backslash B) \cup(B \backslash A) \in \Delta .
$$

It seems natural to conjecture that Th. 2.3 can be generalized to all (finite) difference-closed logics. However, this is not the case.

Example 2.5. Let $X=\{0,1,2, \ldots, 9\}$. Consider the smallest concrete logic $L$ of subsets of $X$ which is difference-closed and contains the sets $A=$ $\{0,1,4,7\}, \quad B=\{0,2,5,8\}, \quad C=\{0,1,2,3\}$ and $D=\{0,4,5,6\}$. (For the reader acquainted with the theory of orthomodular lattices, the logic $L$ is a representation of $M O_{15}$ (thus, difference-closed logics can be lattices without being Boolean). The logic $L$ has 15 mutually non-disjoint elements consisting of 4 points and 15 elements consisting of 6 points that constitute the complements of the former ones).

Let $s$ be a state on $L$ such that $s(A)=s(C)=s(A \delta B)=1$ and $s(B)=$ $s(D)=s(C \delta D)=0$ (there obviously are such states). Let us show that $s$ cannot be extended as a signed state on $\exp X$.

Looking for a contradiction, suppose that $m$ is such an extension. Since $A \cap B=C \cap D=\{0\}$, we obtain

$$
2 m(\{0\})=s(A)+s(B)-s(A \delta B)=0,
$$

and, also,

$$
2 m(\{0\})=s(C)+s(D)-s(C \delta D)=1 .
$$

We have reached a contradiction, verifying the required property.

When we find ourselves within the area of difference-closed concrete logics, it seems of interest to deal with rather special states.

Definition 2.6. Let $L$ be a difference-closed concrete logic on a set $X$ and let $s$ : $\Delta \rightarrow[0,1]$ be a state on $\Delta$. We say that $s$ is subadditive if $s(A \delta B) \leq s(A)+s(B)$ for any pair $A, B \in \Delta$.

The following result indicates the meaning of subadditivity in our context.

Theorem 2.2. Let $X$ be a finite set and let $L=(X, \Delta)$ be a difference-closed concrete logic. Let s be a state on $\Delta$ which allows for an extension, $m$, over $\exp X$ as a signed measure. Let the intersections of pairs of sets of $L$ generate all atoms of a Boolean algebra on $X$. Then $s$ is subadditive if and only if $m$ is a state.

Proof: Compute $s(A)+s(B)-s(A \delta B)$. We obtain

$$
s(A)+s(B)-s(A \delta B)
$$




$$
\begin{aligned}
& =m(A \backslash B)+m(A \cap B)+m(B \backslash A)+m(A \cap B)-m(A \backslash B)-m(B \backslash A) \\
& =2 m(A \cap B) .
\end{aligned}
$$

We therefore see that $m(A \cap B)$ is nonnegative precisely when $s(A)+s(B)-$ $s(A \delta B)$ is nonnegative, which occurs precisely when $s$ is subadditive. We see that the extension $m$ is a state on the Boolean algebra generated by $\Delta$. By the classical theorem, $m$ can be further extended over $\exp X$ as a measure. The proof is complete.

Getting back to Th. 2.3 interplayed with Th. 2.8 , we obtain the following corollary.

Theorem 2.3. Let $X$ be a set of even cardinality. Let $s$ be a state on $X_{\text {even }}$. Then $s$ can be extended over $\exp X$ as a state if and only if $s$ is subadditive.

A natural question arises if a difference-closed logic allows for extensions of states over $\exp X$ as signed measures. Regretfully, it is not the case. We demonstrate it in our final result.

Example 2.7. Let us again consider the logic $L$ of the previous Ex. 2.6. Let us consider the state $s$ defined by setting

$$
\begin{aligned}
& s(A)=\frac{1}{3}, \quad s(B)=\frac{1}{4}, \quad s(C)=\frac{2}{5}, \quad \text { and } \\
& s(Y)=\frac{1}{2} \quad \text { for any } \quad Y \notin\left\{A, B, C, A^{c}, B^{c}, C^{c}\right\} .
\end{aligned}
$$

It is not difficult to check that $s$ is a subadditive state on $L$. This state $s$ cannot be extended over $\exp X$ as a signed measure. Indeed, let $m$ be such an extension. Then

$$
\begin{aligned}
2 m(\{0\}) & =m(A)+m(B)-m(A \delta B) \\
& =s(A)+s(B)-s(A \delta B)=\frac{1}{3}+\frac{1}{4}-\frac{1}{2}=\frac{1}{12},
\end{aligned}
$$

whereas, analogously,

$$
\begin{aligned}
2 m(\{0\}) & =m(C)+m(D)-m(C \delta D) \\
& =s(C)+s(D)-s(C \delta D)=\frac{2}{5}+\frac{1}{2}-\frac{1}{2}=\frac{2}{5} .
\end{aligned}
$$

This is a contradiction. 


\section{ACKNOWLEDGMENTS}

The first author acknowledges the support by the European Union under project Miracle ICA 1- CT-2000-70002 and GNAMPA of INdAM. The second author is grateful to the grant 201/03/0455 of the Czech Science Foundation. The third author acknowledges the support by the Czech Ministry of Education under project MSM 6840770012.

\section{REFERENCES}

Bhaskara Rao, K.P.S. and Bhaskara Rao, M. (1983). Theory of Charges. Academic Press.

De Simone, A. (2000). Absolute continuity of states on concrete logics. International Journal of Theoretical Physics 39(3), 615-620.

De Simone, A. and Pták, P. (2006). Extending coarse-grained measures. Bulletin of the Polish Academy of Sciences Mathematics 54(1), 1-11.

Gudder, S. (1979). Stochastic Methods in Quantum Mechanics. North Holland, New York.

Gudder, S. and Marchand, J. P. (1980). A coarse-grained measure theory. Bulletin de L'Academie Polonaise des Sciences 28(11-12), 557-564.

Horn, A. and Tarski, A. (1948). Measures in Boolean algebras. Transactions of the American Mathematical Society 64, 467-497.

Navara, M. (1989). When is the integration on quantum probability spaces additive? Real Analysis Exchange 14(1), 228-234.

Navara, M. and Pták, P. (1983). Two-valued measures on $\sigma$-classes. Čas. Pěst. Mat. 108, 225-229.

Navara, M. and Pták, P. (1989). Almost Boolean orthomodular posets. Journal of Pure and Applied Algebra 60, 105-111.

Ovchinnikov, P. G. (1999). Measures on finite concrete logics. Proceedings of AMS 127(7), 1957-1966.

Ovchinnikov, P. G. (1992). Measures on the Gudder-Marchand logics (Russian). Constructive Theory of Functions and Functional Analysis 8, 95-98.

Ovchinnikov, P. G. and Sultanbekov, F. (1998). Finite concrete logics: their structure and measures on them. International Journal of Theoretical Physics 1, 147-153.

Prather, R. E. (1980). Generating the $k$-subsets of an $n$-set. American Mathematical Monthly 87, 740-743.

Pták, P. (2000). Concrete quantum logics. International Journal of Theoretical Physics 39, 827-839.

Pták, P. and Pulmannová, S. (1991). Orthomodular Structures as Quantum Logics. Kluwer, Dordrecht/Boston/London.

Sherstnev, A. (1968). On Boolean logics. UZ Kazan GU 128, 48-64 (Russian).

Sultanbekov, F. (1992). Signed measures and automorphisms of a class of finite concrete logics. Constructive Theory of Functions and Functional Analysis 8, 57-68 (Russian).

Svozil, K. (1998). Quantum Logic. Springer, Berlin/Heidelberg. 